NASA Technical Memorandum 100589

\title{
COMPUTATIONAL ASPECTS OF SENSITIVITY CALCULATIONS IN TRANSIENT STRUCTURAL ANALYSIS
}

(NASA-TM-100589) COMPUTATIONAL ASPECTS OF SEN SITIVITY CALCULATICNS IN TRANSIENT STRUCTURAL ANALYSIS (NASA) $37 \mathrm{p}$ CSCL $20 \mathrm{~K}$

\section{G3/39 0136059}

William H. Greene and Raphael T. Haftka

April 1988

\section{N/SA \\ National Aeronautics and Space Administration \\ Langley Research Center Hampton, Virginia 23665}




\title{
COMPUTATIONAL ASPECTS OF SENSITIVITY CALCULATIONS IN TRANSIENT STRUCTURAL ANALYSIS
}

\author{
William H. Greene* \\ NASA Langley Research Center, Hampton, VA 23665 \\ and \\ Raphael T. Haftka $\dagger$ \\ Virginia Polytechnic Institute and State University, Blacksburg, VA 24061
}

\begin{abstract}
A key step in the application of formal automated design techniques to structures under transient loading is the calculation of sensitivities of response quantities to the design parameters. This paper considers structures with general forms of damping acted on by general transient loading and addresses issues of computational errors and computational efficiency. The equations of motion are reduced using the traditional basis of vibration modes and then integrated using a highly accurate, explicit integration technique. A critical point constraint formulation is used to place constraints on the magnitude of each response quantity as a function of time. Three different techniques for calculating sensitivities of the critical point constraints are presented. The first two techniques are based on the straightforward application of the forward and central difference operators, respectively. The third technique is based on explicit differentiation of the equations of motion. Condition errors, finite difference truncation errors, and modal convergence errors for the three techniques are compared by applying them to a simple five-span-beam problem. Sensitivity results are presented for two different transient loading conditions and for both damped and undamped cases.
\end{abstract}

\footnotetext{
* Aerospace Engineer, Structures and Dynamics Division.

† Professor, Department of Aerospace and Ocean Engineering.
} 


\section{Nomenclature}

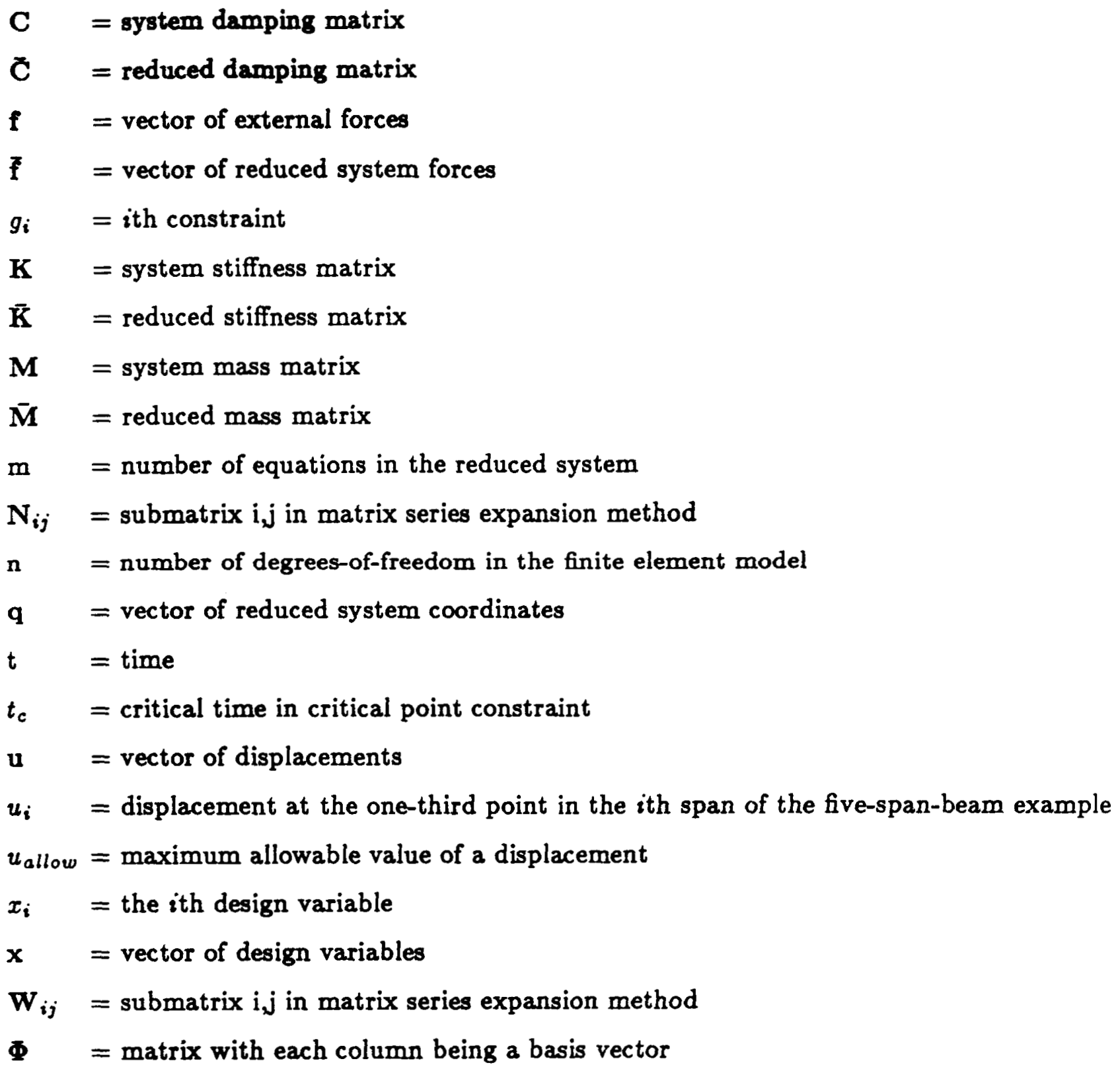

\section{Introduction}

A key step in most automated structural design approaches is the calculation of derivatives of the structural behavior with respect to the design variables (i.e. sensitivities). This problem has been addressed in references describing overall automated design procedures and in references which focus on procedures for sensitivity calculations. Reference [1] provides a comprehensive review of work on calculating sensitivities in structural systems. 
Most of this work has addressed static structural response and structural behavior governed by eigenvalue problems, but a few researchers have considered the problem of sensitivity calculations and optimization in transient structural dynamics problems. Reference [2] considers the minimum mass design of structures under displacement and stress constraints but assumes a simplified form of external, transient loading in order to eliminate the time dependence. Reference [3] considers only harmonic loading but concentrates on the difficult problem of the disjoint design space that often occurs in the automated design problem under dynamic loading.

Another class of work has considered the application of general sensitivity theory to the problem of dynamic mechanical systems under arbitrary loading. Reference [1] summarizes this work and describes three basic approaches which have been employed. In the first method, called the direct method, the transient equations are directly differentiated and solved. In the second method, called the adjoint method, derivatives of a constraint functional are obtained with the aid of an adjoint variable. In the third method, called the Green's function method, the derivatives are obtained in terms of the Green's function of the equations of motion. Although the results from all three methods are theoretically identical, their relative computational efficiency depends on the relative numbers of design variables, degrees of freedom, and constraints. Haug and his co-workers have made considerable progress in developing the adjoint variable method and applying it to problems in machine design and elastic structural design (e.g. refs. [4], [5]). The machine design problems often have the additional complexity of nonlinear equations of motion. However most of these example problems have involved few degrees-of-freedom, design variables, or constraints. There has been little application of these transient sensitivity calculation methods to large-order structural design problems where computational issues become especially important.

The objective of this paper is to address these computational issues in calculating sensitivities in transient structural dynamics problems. In addition to the analytically-based sensitivity formulations described above, straightforward finite difference procedures are also strong candidates for use in transient structural problems. Both a single-step, forward difference procedure and a two-step, central difference procedure are considered herein. A 
version of the direct analytical method described above is also considered. This method uses the directly differentiated transient equations but calculates the derivatives of the coefficients in the transient equations using a forward difference operator. As a consequence, the method is termed semi-analytical. In addition to the selection of sensitivity calculation method, several other computational issues are addressed herein. The first issue concerns numerical solution of the transient equations and the sensitivity equations in the semianalytical method. Any numerical error associated with this solution process could have a significant effect on the accuracy of derivatives. A second issue is the particular constraint formulation used to represent the time-dependent constraints. Herein, a formulation is used where constraints are enforced only at a few, selected, critical points in time. A procedure for selecting these critical times is developed. A third issue concerns the selection of finite difference step size on the accuracy of derivatives. This is a key issue whenever finite difference operators are used and is important in all three sensitivity methods considered herein. A fourth issue is the convergence of the modal method, traditionally used to reduce the computational cost in large-order, linear dynamics problems, as a function of the number of modes used. The particular concern here is the convergence of the sensitivities. Finally, the last issue is the computational cost of the sensitivity calculation methods. This is discussed in general, relative terms rather than with specific performance measures.

The investigation of the above issues is carried out by applying the two finite difference methods and the semi-analytical method to a five-span-beam example. In some cases, concentrated, point masses and dampers are added to the beam. Two different loading conditions are also considered.

\section{Equations of Motion and Solution}

\section{Governing Equations}

The equations of motion for a damped structural system can be written as

$$
\mathbf{M} \ddot{\mathbf{u}}+\mathbf{C u}+\mathbf{K} \mathbf{u}=\mathbf{f}(t)
$$


which is a set of $n$, coupled differential equations. These equations are usually of high order and are typically stiff. Therefore integration techniques used to solve them are typically unconditionally stable and produce an accurate solution only for the low frequency components. The well-known Newmark method (see for example ref. [6]) is an example of such an integration technique.

An alternate approach to the direct integration of eq. (1) is to express the solution in terms of $m$ basis functions

$$
\mathbf{u}=\boldsymbol{\Phi} \mathbf{q}
$$

where $m$ is usually much less than $n$. Then a reduced set of equations can be written

$$
\overline{\mathbf{M}} \ddot{\mathbf{q}}+\overline{\mathbf{C}} \dot{\mathbf{q}}+\overline{\mathbf{K}} \mathbf{q}=\overline{\mathbf{f}}(t)
$$

where $\overline{\mathbf{M}}=\Phi^{T} \mathbf{M} \Phi, \overline{\mathbf{C}}=\Phi^{T} \mathbf{C} \Phi, \overline{\mathbf{K}}=\Phi^{T} \mathbf{K} \Phi$, and $\overline{\mathbf{f}}=\Phi^{T} \mathbf{f}$.

When the undamped natural vibration modes of the system are used as basis functions and $\mathbf{C}$ is a linear combination of $\mathbf{M}$ and $\mathbf{K}$, equation (3) represents a set of uncoupled differential equations which can be solved independently. For certain forms of external loading, such as a piecewise linear approximation, an exact, explicit solution equation is available. This approach is described in [7] and is used in NASTRAN [8].

When vectors which are not the vibration mode shapes are used as basis functions, or a more general form of damping is modeled, the dynamic equations are coupled and must be solved simultaneously. In contrast to eqs. (1), however, eqs. (3) are low order, not stiff, and the primary concern is accurately integrating every equation in the system. Therefore an integration method which minimizes truncation errors in the solution is preferred even if it is only conditionally stable. Accuracy is especially important in sensitivity analyses because errors in the solution process are usually magnified in the calculation of derivatives. Eliminating the integration error due to time step selection can significantly improve the accuracy of sensitivities. 


\section{Solution Method}

An approach that allows the use of moderately large time steps and effectively eliminates truncation error is called the matrix series expansion method in [9] and the transfer matrix method in [10] and [11]. This method expands the solution in a Taylor series where the number of terms determines the accuracy of the approximation. Using this series an expression can be written for the solution at time $t+\Delta t$ in terms of the solution and load at time $t$.

$$
\left\{\begin{array}{c}
\mathbf{q}(t+\Delta t) \\
\dot{\mathbf{q}}(t+\Delta t)
\end{array}\right\}=\left[\begin{array}{ll}
\mathbf{W}_{11} & \mathbf{W}_{12} \\
\mathbf{W}_{21} & \mathbf{W}_{22}
\end{array}\right]\left\{\begin{array}{c}
\mathbf{q}(t) \\
\dot{\mathbf{q}}(t)
\end{array}\right\}+\left[\begin{array}{ll}
\mathbf{N}_{11} & \mathbf{N}_{12} \\
\mathbf{N}_{21} & \mathbf{N}_{22}
\end{array}\right]\left\{\begin{array}{c}
\mathbf{f}(t) \\
\dot{\mathbf{f}}(t)
\end{array}\right\}
$$

It has been assumed here that the load $f$ is approximated as a piecewise linear function of time and therefore the second and higher order derivatives equal zero. The values of the coefficients $\mathbf{W}_{i j}$ and $\mathbf{N}_{i j}$ depend on the number of terms taken in the series. Since the number of terms in the series has only a very small effect on the computational cost of the method, 50 terms were used in this study to effectively eliminate truncation error. Another consideration is that the matrix series expansion method is only conditionally stable and therefore a critical value of $\Delta t$ exists. Numerical experimentation has shown that $\Delta t$ must be smaller than the shortest period of the system to insure numerical stability. Unconditionally stable, low-order methods such as the Newmark method would require much smaller time steps for comparable accuracy.

\section{Critical Point Constraint}

\section{Constraint Formulation}

The general form of the constraint equation is

$$
g_{i}(\mathbf{x}, t) \leq 0
$$

where $\mathbf{x}$ is a vector of design variables and $t$ is time. An effective approach for insuring that this constraint is satisfied for all values of $t$ is the "critical point constraint" approach described in ref. [12] pages 168-169. In this approach a set of peak values of the function 
$g$ (denoted critical points) is selected. An obvious point to include is the time with the "worst" value of $g$. However, if only this point is included, an optimization process modifying a structure based on this information might unknowingly produce a design where the constraint is violated at another time point. To guard against this possibility, a number of important peaks are selected. References [13] and [14] consider in detail the efficient location of critical points in large-scale structures problems with many constraints. The present paper presents a method for selecting the most important peaks as critical points

In the work reported herein, constraints are placed on selected displacements such that

$$
\left|u_{i}(\mathbf{x}, t)\right| \leq u_{\text {allow }}
$$

where $u_{i}$ are the displacements at specific points in the structure and $u_{\text {allow }}$ is the allowable value of the displacement. The critical values of this constraint occur at points in time where $u_{i}$ has the largest magnitude. These are identified by examining every value of $u$ along the time history. In the implementation here, each constraint is assumed to have a specified number of critical points; five critical points for each $u_{i}$ are selected. Values of $u$ where $d u / d t=0$ or values of $u$ at the end points of the time interval are local maxima of $g$ and are termed candidate critical points.

\section{Selection of Critical Points}

The procedure for selecting the critical points from these candidates can best be explained by referring to an example displacement time history shown in figure 1 . The critical points are labeled with circled numbers and a few of the many candidate critical points are labeled with circled letters. The selection criteria applied to every candidate critical point will be explained by considering these few candidate points. Candidate critical points a and c were discarded because the absolute values of the displacements at these points were smaller than those at the five other critical points. The criterion for discarding candidate points $\mathrm{b}$, $\mathrm{d}$, and $\mathrm{e}$ is slightly more complicated. From figure 1 it can be seen that all three candidate points have larger displacement magnitudes than that of critical point 1 , for example. However, candidate points $b$, $d$, and e are all part of "major" peaks where a critical point is selected. A second criterion applied to the selection 
process is a requirement that only one critical point from each major peal be selected. This insures that the critical points represent the total dynamic response rather than just the high frequency undulations on, at worst, a single major peak.

A major peak is identified with the following procedure. Whenever a critical point is selected after comparing its magnitude with that at other critical points, a special screening process is activated. This screening process tests the displacement at every subsequent time

point to determine if it differs from that at this last selected critical point by at least a specified percentage ( $25 \%$ for the studies reported herein). If so, all subsequent time points are no longer considered part of the current major peak. Any candidate critical points identified while this special screening process is in effect are compared only against the last selected critical point.

An example is the major peak in figure 1 which contains points $d$ and 4 . In the selection process, $d$ is initially selected as a critical point and the screening process is activated. The three points where $d u / d t=0$ between point $d$ and point 4 are recognized to be part of the same major peak as d, but since the magnitude of the displacements at these points is smaller than at point $d$, they are discarded. Point 4 is also part of the same major peak as $d$ but since the displacement magnitude there is larger than at point $d$, it replaces $d$ as a critical point. Before the next candidate critical point is considered, the displacement has changed from that at point 4 by more than $25 \%$ and so is considered to be on a new major peak.

\section{Derivatives of Critical Point Constraints}

Once the critical points have been identified for the nominal design, these can used in calculating sensitivities. Reference [12] demonstrates that the change in time location of critical points can be neglected in calculating derivatives of peak values with respect to design variables by examining the expression for the total derivative of $g$ with respect to a design variable $x$. Considering a constraint $g(x, t)$ at a critical time $t_{c}$

$$
\frac{d g\left(x, t_{c}\right)}{d x}=\frac{\partial g}{\partial x}+\frac{\partial g}{\partial t} \frac{d t_{c}}{d x}
$$


The last term in (7) is always zero because at interior critical points $\partial g / \partial t=0$ and on the boundary $d t_{c} / d x=0$. Accordingly, the sensitivity calculations need to be performed only at the specific times where the critical points have been identified. This can result in a considerable savings in computational time, especially when there are many constraints, many time points, or many basis functions used to represent the response. The details of each sensitivity calculation method are discussed below.

\section{Methods For Calculating Sensitivities}

\section{Finite Difference Methods}

Both the forward difference and central difference methods have been used in this study to calculate sensitivities. The well known forward difference approximation to $d u / d x$,

$$
\frac{\Delta u}{\Delta x}=\frac{u(x+\Delta x)-u(x)}{\Delta x}
$$

and central difference approximation

$$
\frac{\Delta u}{\Delta x}=\frac{u(x+\Delta x)-u(x-\Delta x)}{2 \Delta x}
$$

are used. The truncation error for the forward difference approximation is

$$
e_{T}(\Delta x)=\frac{\Delta x}{2} \frac{d^{2} u}{d x^{2}}(x+\varsigma \Delta x) \quad 0 \leq \varsigma \leq 1
$$

and is

$$
e_{T}(\Delta x)=\frac{(\Delta x)^{2}}{6} \frac{d^{3} u}{d x^{3}}(x+\varsigma \Delta x) \quad 0 \leq \varsigma \leq 1
$$

for the central difference approximation.

In applying equations (8) and (9), the selection of difference step size $\Delta x$ is a concern. Selection of a large step size results in errors in the derivative due to truncation of the operator (eqs. 10 and 11). Selection of a small step size can lead to errors in the derivative due to the limited floating point precision of the computer or algorithmic inaccuracies in calculating $u$ (condition errors). Frequently, no acceptable value exists for $\Delta x$ to produce 
an accurate value of $d u / d x$ considering the conflicting requirements of minimizing truncation and condition errors. Because the truncation error associated with the operator of equation 9 is typically less that that of equation 8 , it is possible to use a larger finite difference step size. The larger $\Delta x$ reduces the condition error from the function evaluations and results in a more accurate value of $d u / d x$. However, the necessity of two function evaluations needed for equation 9 makes the procedure more computationally costly.

For the studies in this paper the natural vibration mode shapes are used as basis functions to represent the transient response. In calculating the response of the perturbed design in equation (8) and the two perturbed designs in equation (9) some computational savings are possible relative to the computations for the initial design.

If the mode shapes for the initial design are used to represent the perturbed design, the cost of re-solving the eigenvalue problem is eliminated. However, the reduced set of equations for the perturbed system must still be formed and $\overline{\mathbf{M}}, \overline{\mathbf{C}}, \overline{\mathbf{K}}$ are now full. This coupled system is then solved using the matrix series expansion method described above.

If the updated mode shapes for the perturbed design are used in the analysis, many eigensolution procedures, such as the subspace iteration used here, can begin with the mode shapes from the initial design as approximations. Since the perturbation in the design is small, the subspace iteration procedure converges rapidly. However, at least one factorization of $\mathbf{K}$ is required. For large finite element models this can be the largest part of the computational cost. For most of the studies in this paper, the forward difference method used the initial mode shapes to represent the perturbed design. Because the central difference method was used for reference values of derivatives, updated mode shapes were calculated for the two required perturbed designs. In both case, because of the critical point constraint formulation, the transformation from modal coordinates to physical coordinates (displacements, stresses, etc.) is performed only at the critical times instead of at all time points. 


\section{Semi-Analytical Method}

The direct method for sensitivity calculation is derived by differentiating equation (3). This is presented in detail in [12] pages 169-171 and will be summarized here. After differentiating (3) with respect to the design variable,$x$, the result is

$$
\overline{\mathbf{M}} \frac{d \ddot{\mathbf{q}}}{d x}+\overline{\mathbf{C}} \frac{d \dot{\mathbf{q}}}{d x}+\overline{\mathbf{K}} \frac{d \mathbf{q}}{d x}=\frac{d \overline{\mathbf{f}}}{d x}-\frac{d \overline{\mathbf{M}}}{d x} \ddot{\mathbf{q}}-\frac{d \overline{\mathbf{C}}}{d x} \dot{\mathbf{q}}-\frac{d \overline{\mathbf{K}}}{d x} \mathbf{q}
$$

The first step in forming this equation is the calculation of the derivatives of $\overline{\mathbf{f}}, \overline{\mathbf{M}}, \overline{\mathbf{C}}$, and $\overline{\mathbf{K}}$ (equation 3 ) with respect to $x$. This process is simplified considerably by using a fixed set of basis functions, $\Phi$. For the studies in this paper $\boldsymbol{\Phi}$ is taken as a set of vibration modes of the initial structure. With $\mathbf{\Phi}$ independent of $x$, differentiation of $\overline{\mathbf{K}}$ yields

$$
\frac{d \overline{\mathbf{K}}}{d x}=\boldsymbol{\Phi}^{T} \frac{d \mathbf{K}}{d x} \boldsymbol{\Phi}
$$

with similar expressions for the derivatives of $\overline{\mathbf{M}}$ and $\overline{\mathbf{C}}$.

The force $f$ is frequently not a function of the design variables (as in the models of this paper) or is an explicit function of $x$ and therefore easy to differentiate. The derivatives of $\mathbf{M}, \mathbf{C}$, and $\mathbf{K}$ are, in general, difficult to calculate and for this reason these derivatives are often replaced by finite difference approximations. The combination of analytical differentiation of the response equations with finite difference matrix derivatives is known as a semi-analytical approach. Semi-analytical methods have become the methods of choice in static, structural sensitivity calculations because they allow generality in the selection of design variables with moderate computational cost. Herein a semi-analytical implementation of the direct method (abbreviated as "the semi-analytical method") is used with the matrix derivatives calculated using the forward difference operator. For several important classes of design variables, however, $\mathbf{M}, \mathbf{C}$, and $\mathbf{K}$ are linear functions of $x$. For example, $\mathbf{M}$ and $\mathbf{K}$ in a finite element model composed of truss members are linear functions of member cross sectional area. In these cases there are no truncation errors and large finite difference step sizes can be used to reduce the condition error and produce accurate derivatives. 
After forming the derivatives of $\overline{\mathbf{f}}, \overline{\mathrm{M}}, \overline{\mathrm{C}}$, and $\overline{\mathbf{K}}$ the right-hand side of (12) can be formed using $\ddot{q}, \dot{q}$, and $q$ from the solution of (3). The matrix series expansion method insures that accurate values of $\ddot{\mathbf{q}}, \dot{q}$, and $q$ are available for this step. . Equation (12) can then be integrated in time to yield $d \ddot{q} / d x, d \dot{q} / d x$, and $d \mathbf{q} / d x$. Again, because of the critical point constraint formulation, the transformation from these modal quantities to physical quantities is performed only at the critical times.

\section{Computational Cost of the Methods}

All three methods require an identical analysis of the initial design. If updated vibration modes are used in the two finite difference methods, this analysis must be repeated resulting in a high computational cost for calculating the sensitivities. For large problems, the cost of re-solving the vibrational eigenproblem for each perturbed case would become excessive. The two methods presented here with reasonable costs for large problems are the forward difference method with fixed modes and the semi-analytical method. The forward difference method requires $\bar{M}, \bar{C}$, and $\overline{\mathbf{K}}$ for the perturbed design. The semianalytical method, however, requires formation of $d \overline{\mathbf{M}} / d x, d \overline{\mathbf{C}} / d x$, and $d \overline{\mathbf{K}} / d x$. The costs for these two operations are roughly the same. The forward difference method requires the integration of the reduced equations of motion (3) for the perturbed model. The semi-analytical method requires integration of (12). The costs for these two operations are similar. The semi-analytical method, however, requires the formation of the righthand-side in eq. (12) and there is no similar operation in the forward difference method. To form the right-hand-side of (12) three matrix multiplications must be performed at each time step. Similar operations are performed in integrating the reduced equations of motion (see eq. 4) so the cost of forming the right-hand-side of (12) is of the same order as an additional time integration. This cost will not become large unless the order of the reduced system, $\mathrm{m}$, or the number of time steps become large. 


\section{Computer Implementation}

The three methods for calculating sensitivities have been implemented using a slightly modified version of the general purpose finite element code, EAL [9]. EAL includes general language constructs for controlling execution flow as well as general and specific utilities for manipulating data stored as named entities in a database. Each method was implemented as a set of EAL procedures which are invoked in a manner similar to FORTRAN subroutines.

The transient response module in EAL version 312 solves the uncoupled form of equation (3) using the matrix series expansion method. A modification was made to allow equation (3) to be fully coupled. In the semi-analytical method, the right-hand side, pseudo loading of equation (12) can be easily formed using EAL. However, a slight modification to the transient response module was required to permit solution of equation (12) with this general form of loading. In addition, a special purpose module was added to EAL to perform the task of identifying the critical points on each response function.

Most of the calculations were performed using a CDC Cyber version of EAL where the floating point operations were done with 60 bit precision. In order to demonstrate the effects of condition error, a few calculations were performed using a DEC VAX version of EAL where most of the floating point operations were done with 32 bit precision.

\section{Five-span-beam Example}

The three methods for calculating sensitivities are applied to a five-span, planar beam example taken from reference [15] and shown in figure 2. The beam is modeled with three beam finite elements per span resulting in 26 unconstrained degrees-of-freedom. As shown in figure 2, translational and rotational viscous dampers were also added to the beam. These devices are representative of velocity feedback controllers which might be added to flexible structures. Cases with and without dampers were considered. The numerical values of the damping coefficients from ref. [15] of $c_{1}=.008 \mathrm{sec}-\mathrm{lbf} /$ in and $c_{2}=1.2 \mathrm{sec}-\mathrm{lbf}$ were used. A case was also considered where a $1.0 \mathrm{lb}$ mass (approximately $20 \%$ of the beam's mass) was added to the beam at the location of the translational damper. The 
Table 1. Eigenvalues For the Three Five-span-beam Cases

\begin{tabular}{|c|c|c|c|c|}
\hline \multirow{2}{*}{ Mode } & Undamped & \multicolumn{2}{|c|}{ Damped } & $\begin{array}{c}\text { Undamped } \\
\text { With Point Mass }\end{array}$ \\
\cline { 2 - 5 } & Frequency, Hz. & Frequency, Hz. & Damping Ratio & Frequency, Hz. \\
\hline \hline 1 & 1.1707 & 1.2210 & .0851 & .9401 \\
2 & 1.2991 & 1.2926 & .0352 & 1.2594 \\
3 & 1.6254 & 1.6298 & .0690 & 1.5445 \\
4 & 2.0491 & 2.0910 & .0590 & 1.8005 \\
5 & 2.4628 & 2.5497 & .0958 & 2.3729 \\
6 & 4.7343 & 4.8426 & .0044 & 4.2327 \\
7 & 5.0105 & 4.9785 & .0413 & 4.8858 \\
8 & 5.6472 & 5.7703 & .0126 & 5.6400 \\
9 & 6.4153 & 6.4178 & .0407 & 5.9261 \\
10 & 7.1274 & 7.2229 & .0193 & 6.8762 \\
\hline
\end{tabular}

eigenvalues for these three cases are shown in table 1. The additional point mass has a significant effect on the frequencies while the dampers have little effect.

\section{Effect of Loading and Damping on Response}

The applied loading for all studies consisted of a point moment of .04405 in-lbs applied at the right end of the beam. Two different time functions for this load, a step and a ramp, (shown in figure 2) were considered. The step load tends to excite many of the higher modes of the beam. This is shown in figure $3 a$ where a selected displacement for the undamped beam, $u_{2}$ (figure 2), is plotted as a function of time. The participation of the higher frequency modes in the response is obvious from the jagged nature of the peaks. However, when the load is applied more gradually with the ramp function shown in figure 2 , the higher modes are excited less. This is shown in figure $3 \mathrm{~b}$ where displacement $u_{2}$ is plotted as a function of time. The addition of the two point dampers also serves to reduce the participation of the high frequency modes for the beam under the step load. Displacement $u_{2}$ is plotted as a function of time in figure $3 c$ for this case. All 26 vibration modes were 
used in the analysis shown in figure 3. The effect on both displacements and sensitivities of including fewer modes for each of the two load cases will be discussed below.

\section{Design Variables}

Two different classes of design variables were considered. The first design variable is a concentrated mass (initially zero) at the location of the translational damper. This design variable was also considered in [15]. The derivatives of the system mass and stiffness matrices with respect to this design variable are constant and zero, respectively. As a consequence, the derivatives of the system matrices required in the semi-analytical method can be calculated exactly by a simple forward difference operator. The beam thicknesses in each of the five spans were also design variables. Derivatives with respect to the five thickness design variables showed similar characteristics. Herein, results for derivatives with respect to the thickness in the rightmost span, $h$, along with derivatives with respect to the point mass, $\mathrm{m}$, are presented.

\section{Effect of Finite Difference Step size on Derivatives}

The three methods described above for calculating sensitivities all rely on finite difference operators at some stage in the algorithm. The forward and central difference methods rely on the operators in equations (8) and (9) to calculate derivatives of response quantities. In the semi-analytical method the derivatives of the system matrices are calculated using the forward difference operator in equation (8). In all three cases the finite difference step size must be selected so that the operator provides a reasonable approximation to the derivative. If the step size is too large, the error due to truncating the series approximation of the derivative is large. If the step size is too small, the numerical condition error in performing the function evaluations (dynamic analyses) becomes large.

To assess the effect of step size on the calculation of sensitivities for the five-spanbeam, derivatives were calculated using the three methods with various step sizes. In this study the beam is undamped and the ramp loading is applied. All 26 vibration modes were included in the analysis. Figure $4 a$ shows the derivative of displacement $u_{2}$ at critical point number 5 with respect to the point mass design variable, $\mathrm{m}$, as a function of step size. 
As mentioned above, the derivatives of the system matrices with respect to this design variable can be calculated exactly in the semi-analytical method. As a result, the semianalytical derivative is approximately constant for the six-order-of-magnitude change in step size shown in the figure. The central difference method uses the higher order operator and provides good accuracy over most of the step size range shown in the figure. The forward difference operator provides good accuracy with the smaller step sizes but begins to diverge earlier than the central difference method for the larger step sizes.

Figure $4 \mathrm{~b}$ shows the derivative of displacement $u_{1}$ at critical point number 5 with respect to the right-most span thickness, $h$, as a function of step size. In this case the system mass matrix is a linear function of this design variable and its derivative can be computed exactly. The system stiffness matrix is a cubic function of this design variable and its derivative can only be approximated by the forward difference operator. Still, the derivative computed by the semi-analytical method is very accurate except for the largest step size and is no worse for this case than the much more costly central difference method. Again, the forward difference operator results in substantial errors for the larger step sizes.

Because this example has a relatively small number of degrees of freedom there is little condition error when small step sizes are used. To assess the effects of condition error which would occur for larger problems, the derivatives for the five-span-beam problem were also calculated on a DEC VAX computer. In the VAX calculations many of the floating point operations are performed using 32 bit precision compared with the 60 bit precision used in the studies described above. The derivative of displacement $u_{2}$ at critical point number 5 with respect to the point mass is plotted as a function of finite difference step size in figure 4c. Derivatives are calculated using the semi-analytical method, the central difference method, and the forward difference method. For the larger step sizes, the results from all four methods agree well with those calculated using 60 bit precision. For step sizes smaller than $10^{-4}$ there is considerable error in the derivatives calculated using the forward and central difference methods. The derivatives calculated using the semi-analytical method, however, are highly accurate over the entire range of step sizes shown in the figure. 


\section{Modal Convergence of Displacements and Derivatives}

When vibration modes or other functions are used to reduce the basis in a transient response problem (equation 2), the key question is how many modes are required for an accurate solution. When the objective of the analysis is the calculation of sensitivities, small errors in the solution due to a reduced set of modes can have a pronounced effect on derivatives.

Figure 5 shows the effect of number of vibration modes used in the analysis of the five-span-beam on selected displacements (shown by subscripts) at selected critical points (in parenthesis). Results for ramp and step loads acting on the undamped beam and the step load acting on the damped beam are shown. The displacement/critical point combinations were selected to be representative of both the largest and smallest critical values. In all cases the convergence is very good with approximately ten modes yielding a converged solution. Although, as shown in figure 3a, there is considerable high frequency participation in the displacement history, the displacements at critical points are dominated by low frequency modes.

The modal convergence of the displacement derivatives is considerably different, however. Selected derivatives with respect to the point mass design variable are shown in figure 6 as a function of number of modes in the analysis. All derivative results shown in the figure were obtained using the central difference method with updated vibration modes to reduce errors associated with the finite difference operator and the modes. The consequences of keeping the vibration modes fixed instead of updating them are discussed below. For the step loading case with no damping, the derivatives of $u_{1}$ and $u_{2}$ show especially poor convergence with more than 20 modes required. Small errors in the solutions for $u_{1}$ and $u_{2}$ due to modal truncation, which are unnoticeable in figure 5 , are being magnified by the differentiation. For the ramp loading case with no damping, where the higher frequency modes play a less important role in the solution (see figure $3 \mathrm{~b}$ ) the convergence of the derivatives is nearly as good as for the displacements.

For the case of the step loading with damping, the convergence of the derivatives is not as good as for the ramp loading case but better than for the case of step loading on 
the undamped beam. The damping appears to improve convergence in the step loaded beam by filtering the high frequency modes from the displacement response.

Figure 7 shows modal convergence results for selected displacement derivatives with respect to the thickness in the right-most span of the beam. The results are similar to those for the derivatives with respect to the point mass. The convergence for the derivatives in the step loaded, undamped beam is also especially slow. Approximately 24 of the total 26 modes are required for converged derivatives. As before, the derivatives for the ramp loaded, undamped beam show good convergence. Convergence of the derivatives for the damped beam under the step load is also acceptable (i.e. convergence was achieved with about 10 modes).

\section{Fixed Versus Updated Modes in Derivative Calculations}

As mentioned above, the computational cost of updating the vibration modes for the perturbed analyses is substantial. The question of whether the modes from an initial design can be used in a finite-difference-based procedure to calculate sensitivities of the transient behavior has received considerable attention in the literature. In ref. [16] it was shown that there is a substantial difference in the derivatives of aircraft flutter speeds when fixed modes are used rather than the updated modes. In ref. [15], however, there was little difference in the derivatives of damping ratios for the five-span-beam when either

fixed or updated modes were used. This was investigated here using the same five-span, undamped beam under the step load. As shown in figures 6 and 7 where the derivatives were calculated using the central difference method with updated modes, convergence with respect to the number of modes is very slow. Figure 8 shows the derivatives of selected displacements with respect to the right-most beam thickness calculated using forward difference procedures. Results with both fixed and updated vibration modes are shown. Again, the convergence as a function of number of modes is poor. However, for all three derivatives, the results are nearly the same for both the fixed mode and updated mode cases.

A final study was performed to assess whether fixed modes can also produce accurate derivatives when the structure is substantially perturbed. For this case the point mass was 
increased from zero to about $20 \%$ of the mass of the beam resulting in substantial frequency changes (see Table 1). The ramp loading was applied and the beam was undamped. The vibration mode shapes used in the forward difference method were calculated for the beam with zero point mass. Derivatives of selected beam displacements plotted as a function of the number of these modes are shown in figure 9. Convergence as a function of number of modes is fairly good in each case indicating that, at least for this example, the fixed modes can be used even after significant changes have been made to the original structure.

\section{Concluding Remarks}

Several computational concerns which arise in calculating the sensitivity of response quantities to design variable changes in structures under transient loading have been considered. The structure can include general forms of damping and be acted on by general, transient loading. Natural vibration mode shapes are used to reduce the basis of the resulting transient problem. A critical point constraint formulation is used where explicit constraints are placed only on the largest values of a response quantity along a time history. Three sensitivity calculation methods are investigated in this study. Two of the methods involve application of the well-known one-point forward difference and two-point central difference operators. A third method involves explicit differentiation of the equations of motion to yield a set of differential equations in the sensitivities. The procedures are denoted forward difference, central difference, and semi-analytical respectively. All three methods use a highly accurate, explicit, time integration technique to reduce numerical errors in the integration of the equations of motion.

The three methods are applied to a five-span damped beam acted on by an applied moment. Both a step and a ramp time distribution of the moment are considered. Two important issues investigated are the effect of finite difference step size on derivative accuracy and the effect of number of included vibration modes on derivative accuracy. The central difference method is relatively insensitive to finite difference step size but is computationally costly because two analyses of perturbed designs are required. Results from the forward difference method were shown to be accurate for small step sizes but in error for larger step sizes. The semi-analytical method, on the other hand, was shown to 
be as insensitive to step size as the central difference method but with a computational cost only slightly greater than the forward difference method. Calculations using all three methods performed on a computer with lower floating point precision indicate that the semi-analytical method may be more insensitive to condition errors than the forward or central difference methods.

The convergence of derivatives from all three methods as a function of number of modes in the analysis is similar. The time distribution of the loading, however, has a significant effect on modal convergence. Even though displacement quantities show good convergence for both the ramp and step loads, the convergence of derivatives for the beam under the step load is poor. For the five-span-beam example, the sensitivities calculated using fixed vibration modes are nearly identical to those calculated using updated modes. Using fixed modes substantially reduces the computational cost of sensitivity calculations. In addition, results from a computer with lower floating point precision indicate a potential improvement in the results for small step sizes when fixed rather than updated modes are used in the calculations. It was shown that damping improves the modal convergence.

Based on insensitivity to finite difference step size, modal convergence, and computational cost, the semi-analytical method appears to be the best choice for calculating sensitivities in transient response problems. 


\section{References}

1. Adelman, Howard M., and Haftka, Raphael T., "Sensitivity Analysis of Discrete Structural Systems." AIAA Journal, Vol. 24, No. 5, May 1986, pp. 823-832.

2. Fox, R. L., and Kapoor, M. P., "Structural Optimization in the Dynamics Regime: A Computational Approach." AIAA Journal, Vol. 8, No. 10, October 1970, pp. 1798-1804.

3. Mills-Curran, W. C., and Schmit, L. A., "Structural Optimization With Dynamic Behavior Constraints." AIAA Journal, Vol. 23, No. 1, January, 1985, pp. 132-138.

4. Haug, Edward J., Arora, Jasbir, S., "Design Sensitivity Analysis of Elastic Mechanical Systems." Computer Methods in Applied Mecahnics and Engineering, Vol. 15, 1978, pp. 35-62.

5. Haug, Edward J., "Design Sensitivity Analysis of Dynamic Systems." NATO ASI Series, Vol F27, Computer Aided Optimal Design: Structural and Mechanical Systems, Edited by C. A. Mota Soares, Springer-Verlag, 1987, 705-755.

6. Bathe, Klaus-Jürgen, Finite Element Procedures in Engineering Analysis. PrenticeHall, Inc., 1982.

7. Craig, Jr., R. R.,Structural Dynamics: An Introduction to Computer Methods. John Wiley \& Sons, Inc., 1981.

8. The NASTRAN Theoretical Manual. NASA SP-221(06), National Aeronautics and Space Administration, January, 1981.

9. Whetstone, W. D., EISI-EAL Engineering Analysis Language Reference Manual EISI-EAL System Level 912. Engineering Information Systems, Inc., August 1985.

10. Brenneman, B., "An Analytic Solution of Systems of Linear Ordinary Differential Equations Useful for Numerical Integration," thesis presented to Virginia Polytechnic Institute and State University, Blacksburg, Va., 1973.

11. Melosh, Robert J., "Integration of Linear Equations of Motion." Journal of The Structural Division, ASCE, Vol. 101, No. ST7, July, 1975, pp. 1551-1558.

12. Haftka, Raphael T., and Kamat, Manohar P., Elements of Structural Optimization. Martinus Nijhoff Publishers, 1985.

13. Grandhi, Ramana V., Haftka, Raphael T., and Watson, Layne T., " Efficient Identification of Critical Stresses in Structures Subject to Dynamic Loads." Computers and Structures, Vol. 22, No. 3, 1986, pp. 373-386.

14. Grandhi, Ramana V., Haftka, Raphael T., and Watson, Layne T., "Design-Oriented Identification of Critical Times in Transient Response." AIAA Journal, Vol. 24, No. 4, April, 1986, pp. 649-656.

15. Sandridge, Chris A., and Haftka, Raphael, T., "Accuracy of Derivatives of Control Performance Using A Reduced Structural Model." Presented AIAA/ASME/AHS/ASEE 
28th Structures, Structural Dynamics and Materials Conference, Monterey, CA, April 6-8, 1987.

16. Haftka, Raphael T., and Yates Jr., E. Carson, "Repetitive Flutter Calculations in Structural Design." Journal of Aircraft, Vol. 13, No. 7, July, 1976, pp. 454-461. 

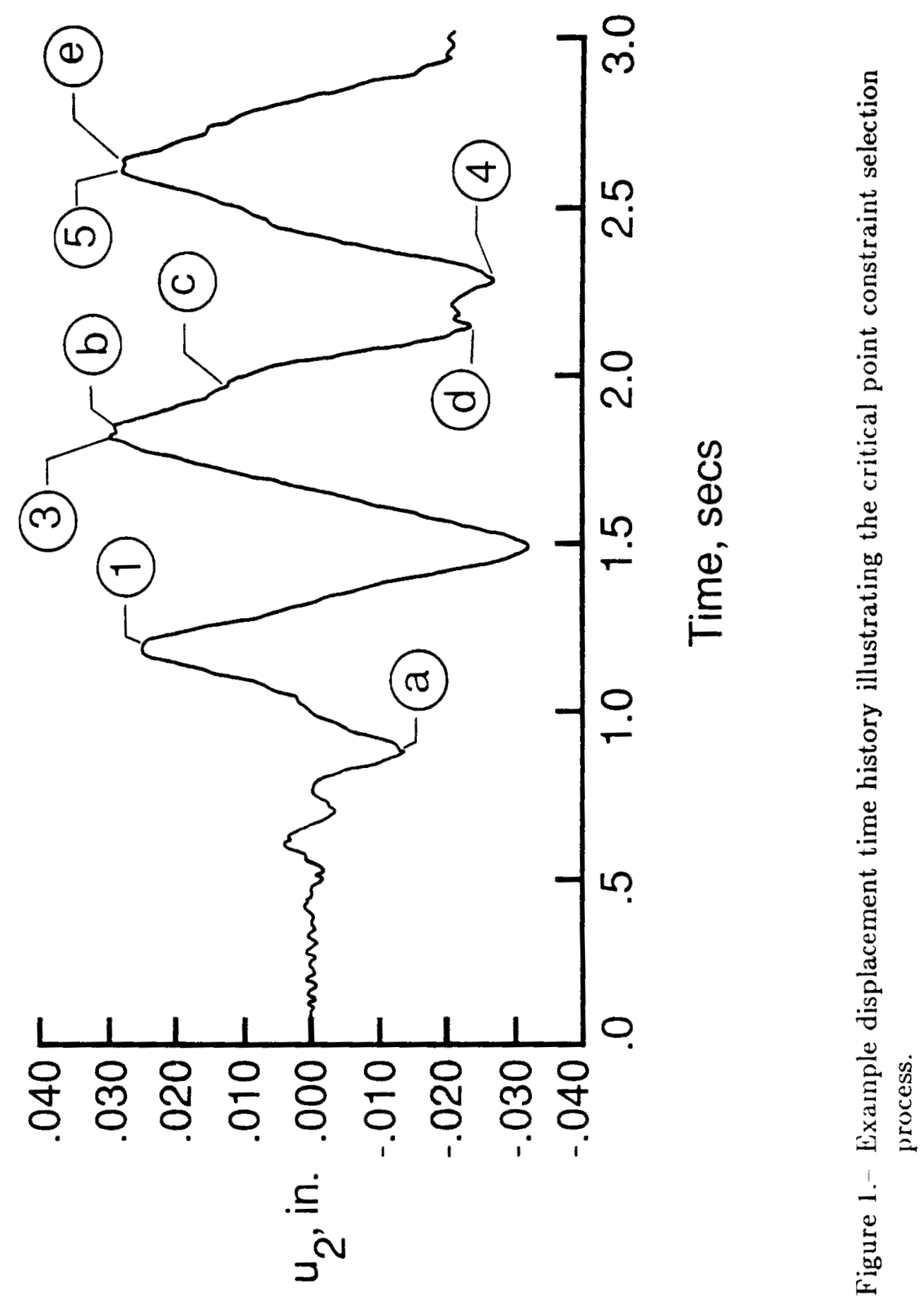


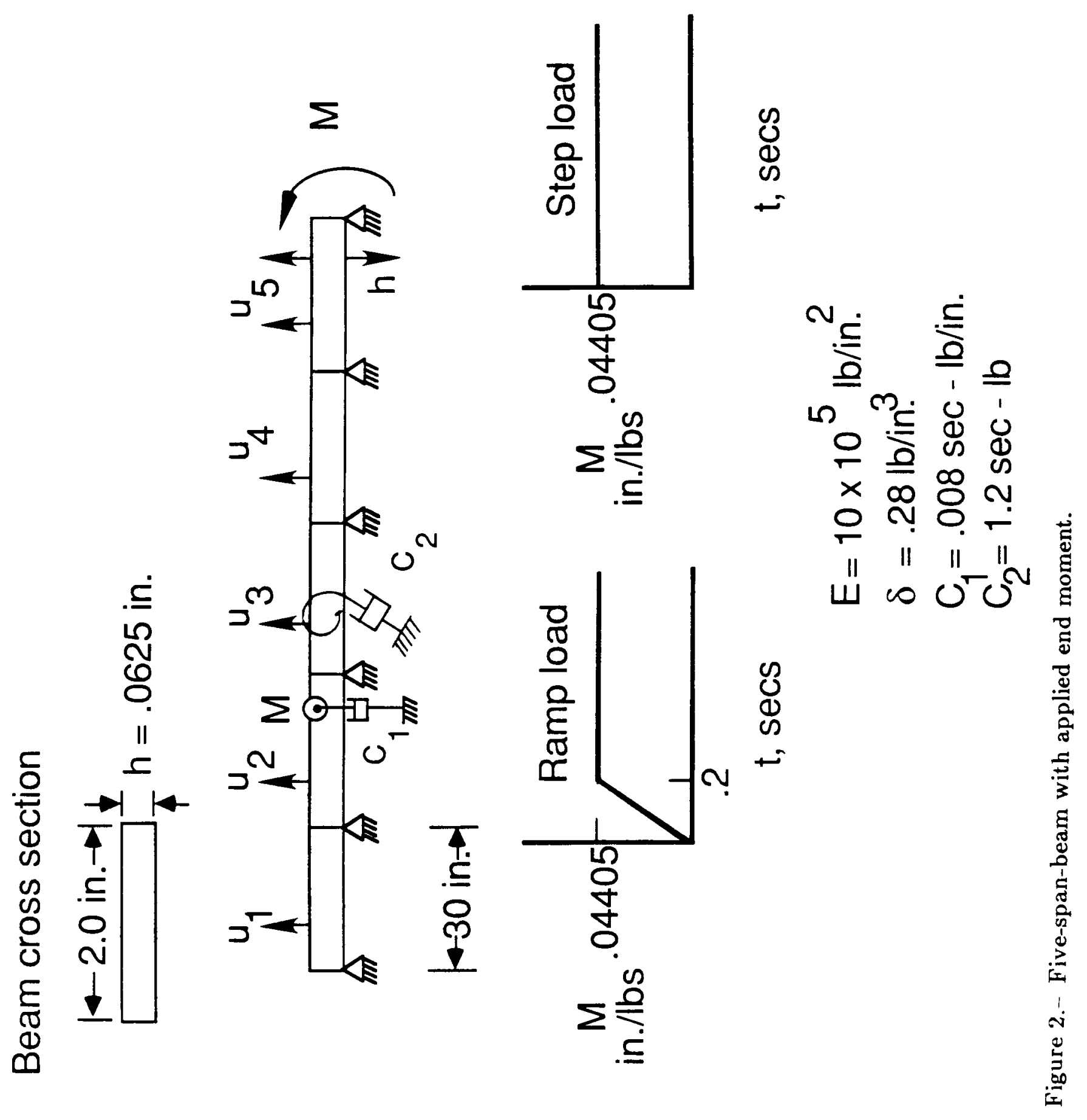



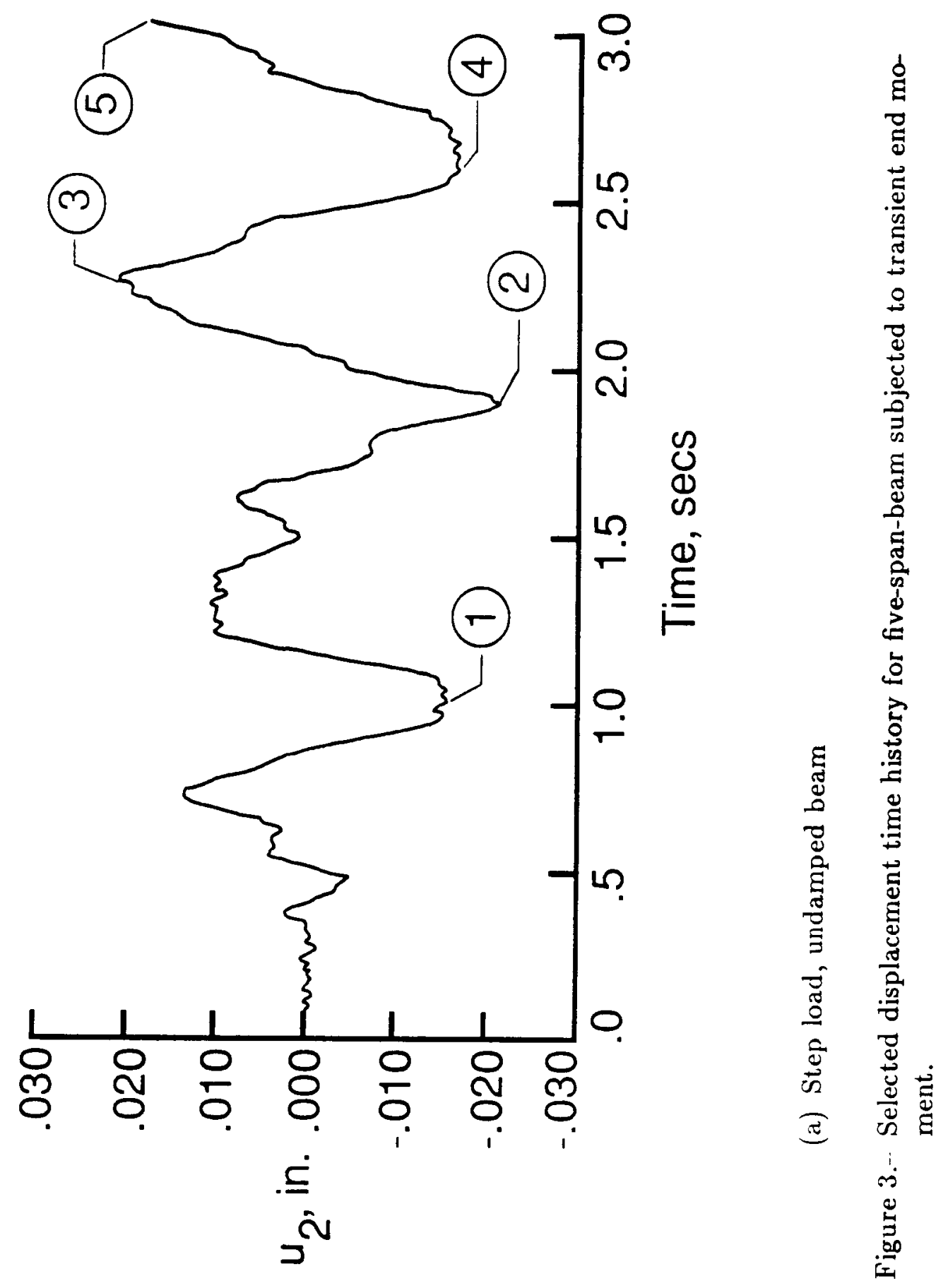

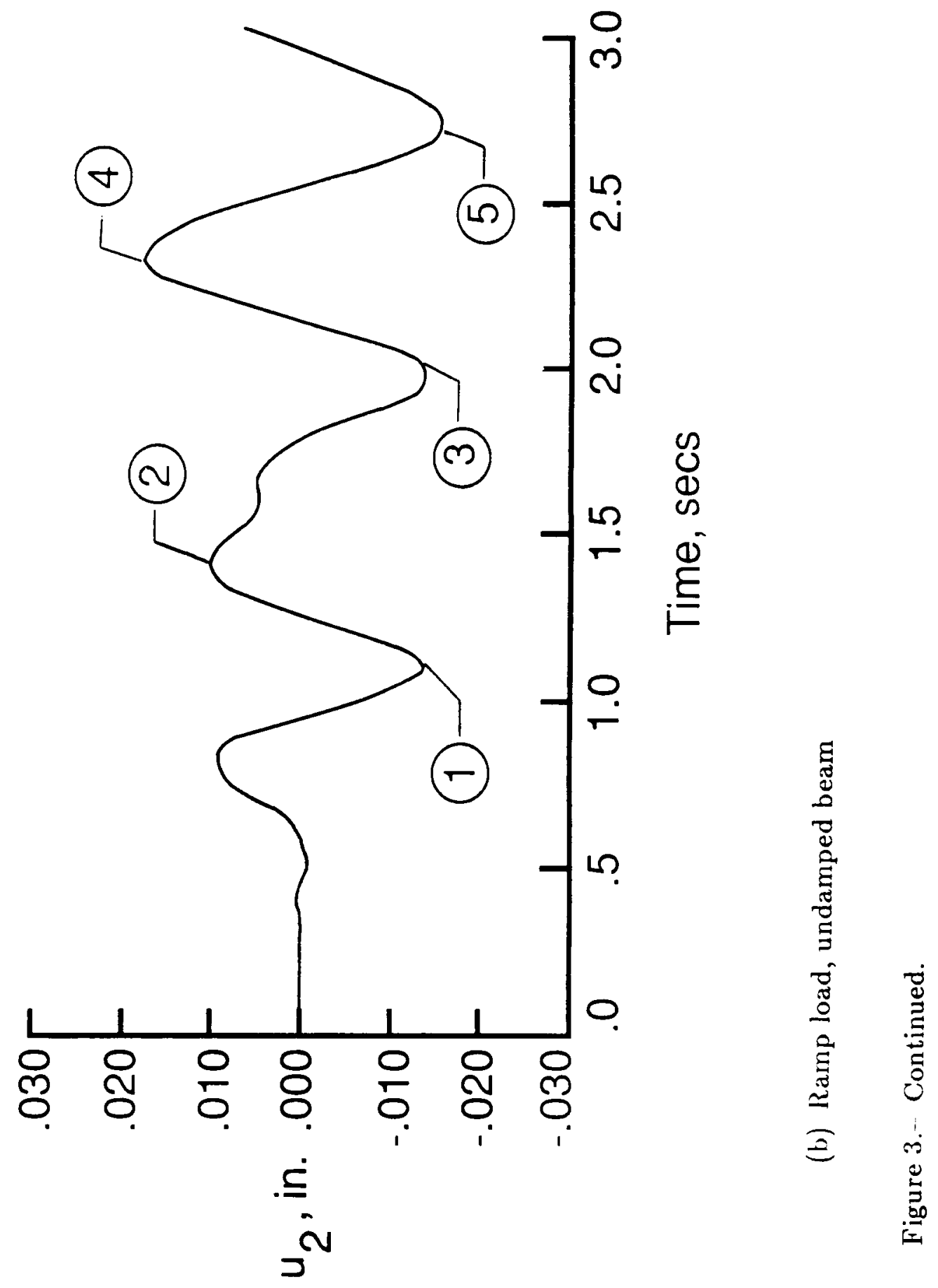

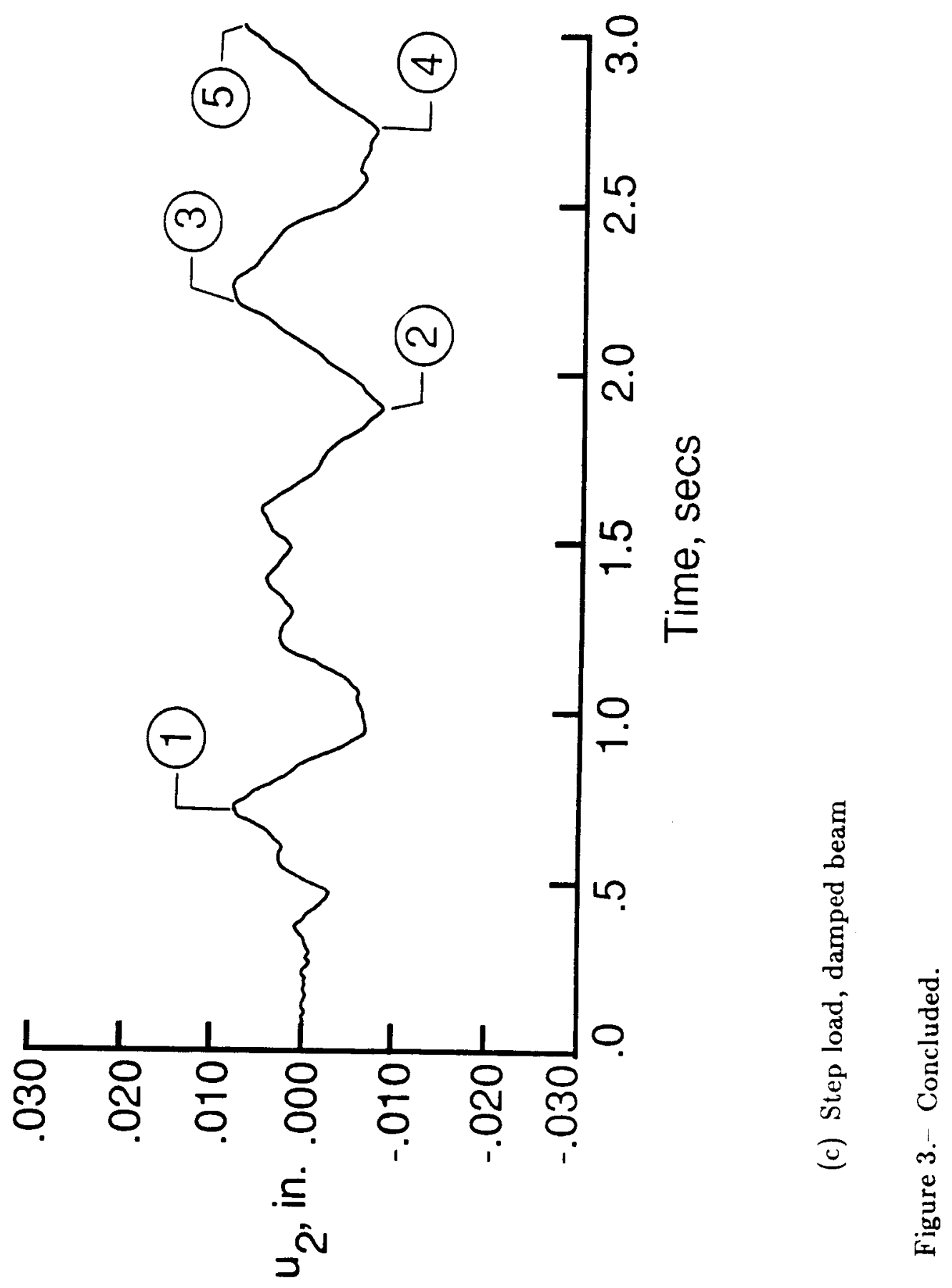


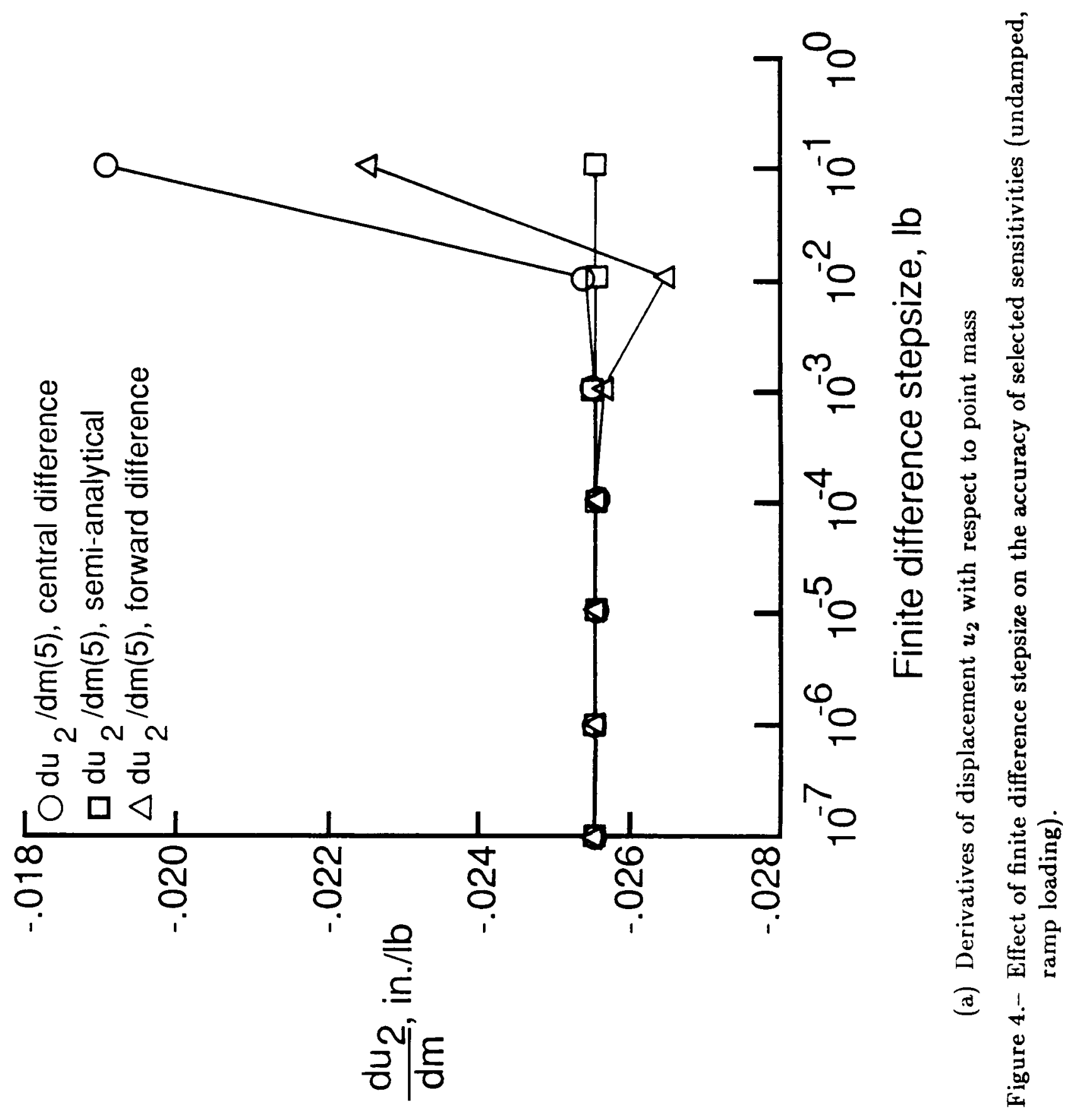




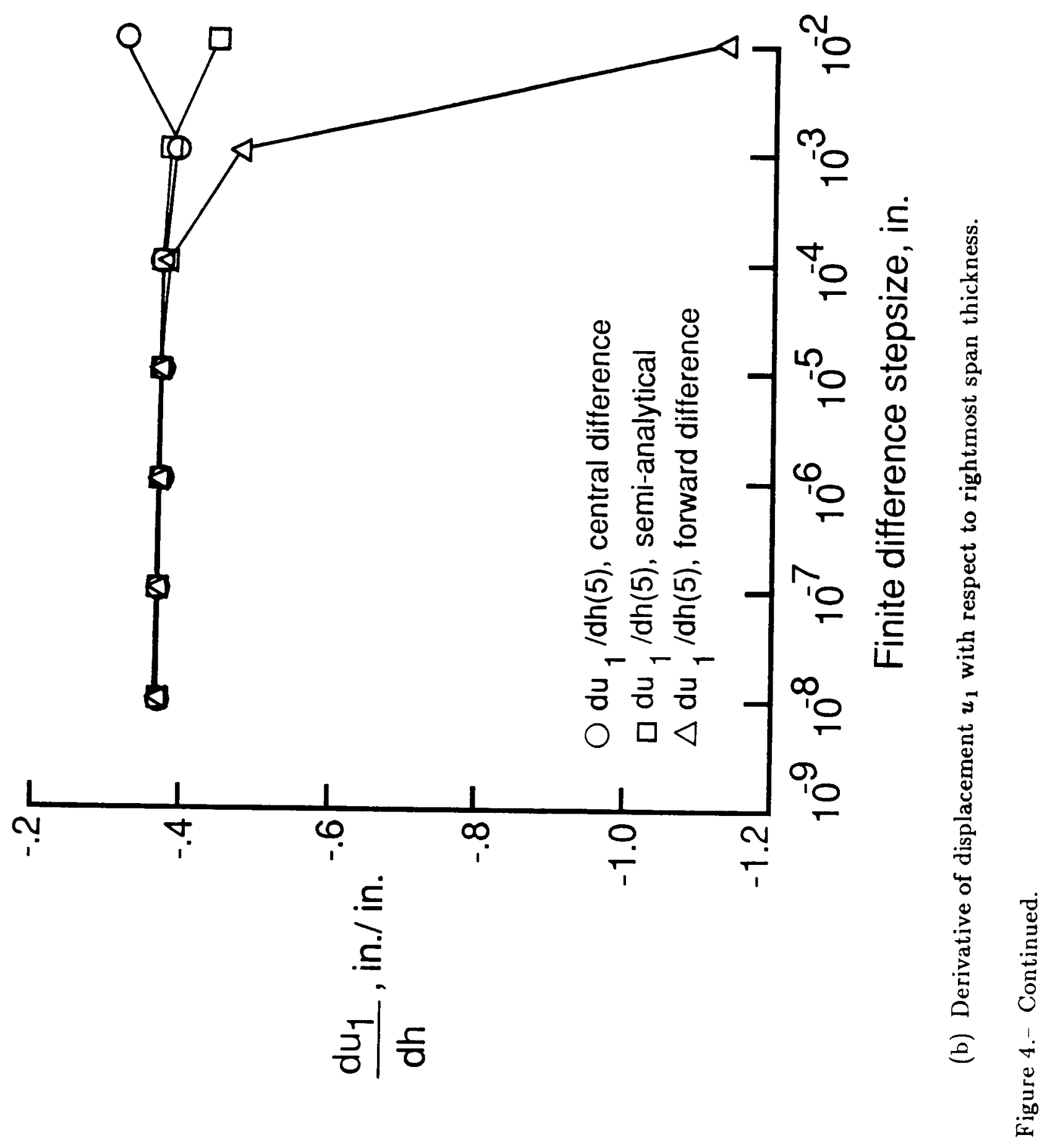



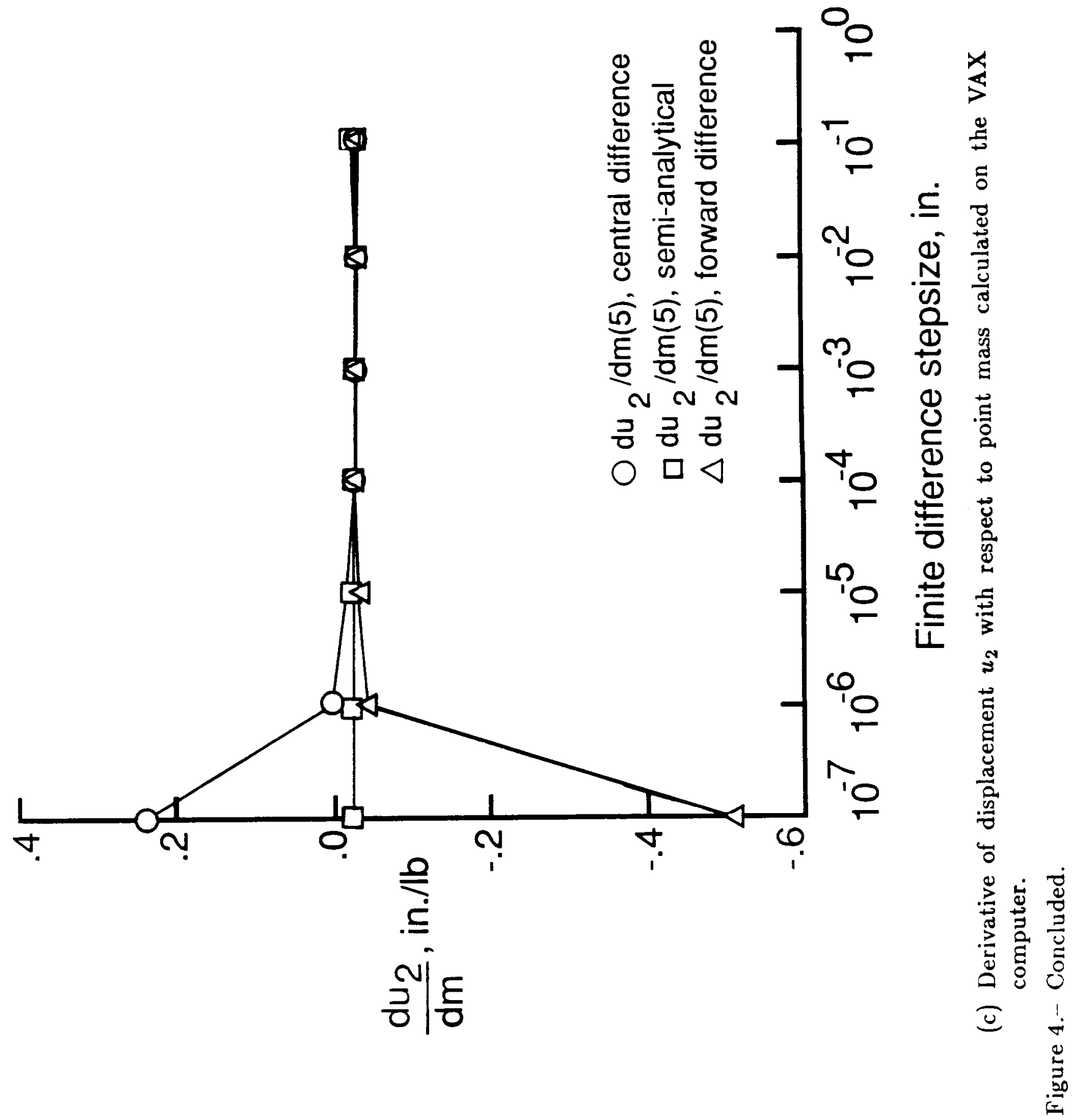


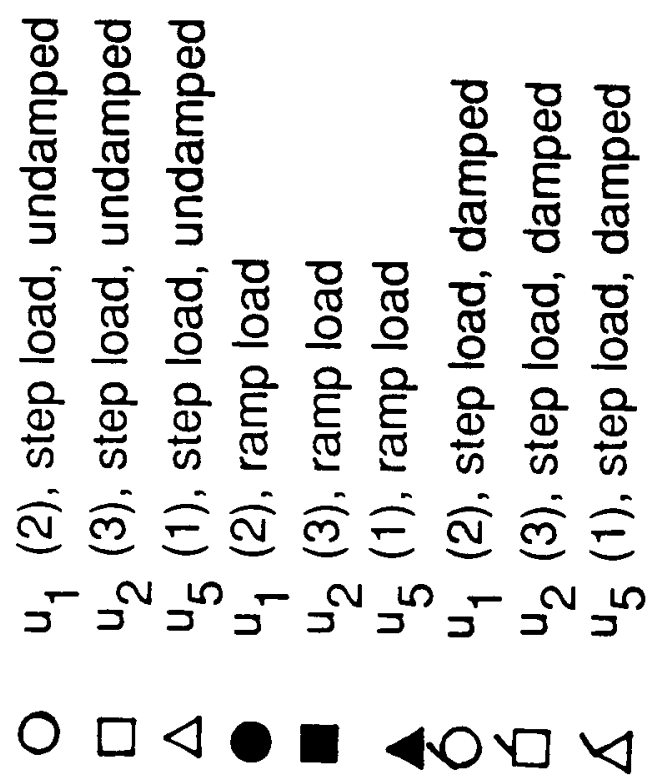

$\bigcirc \square \triangleleft \bullet \square \triangleleft 0 \vee \triangleleft$

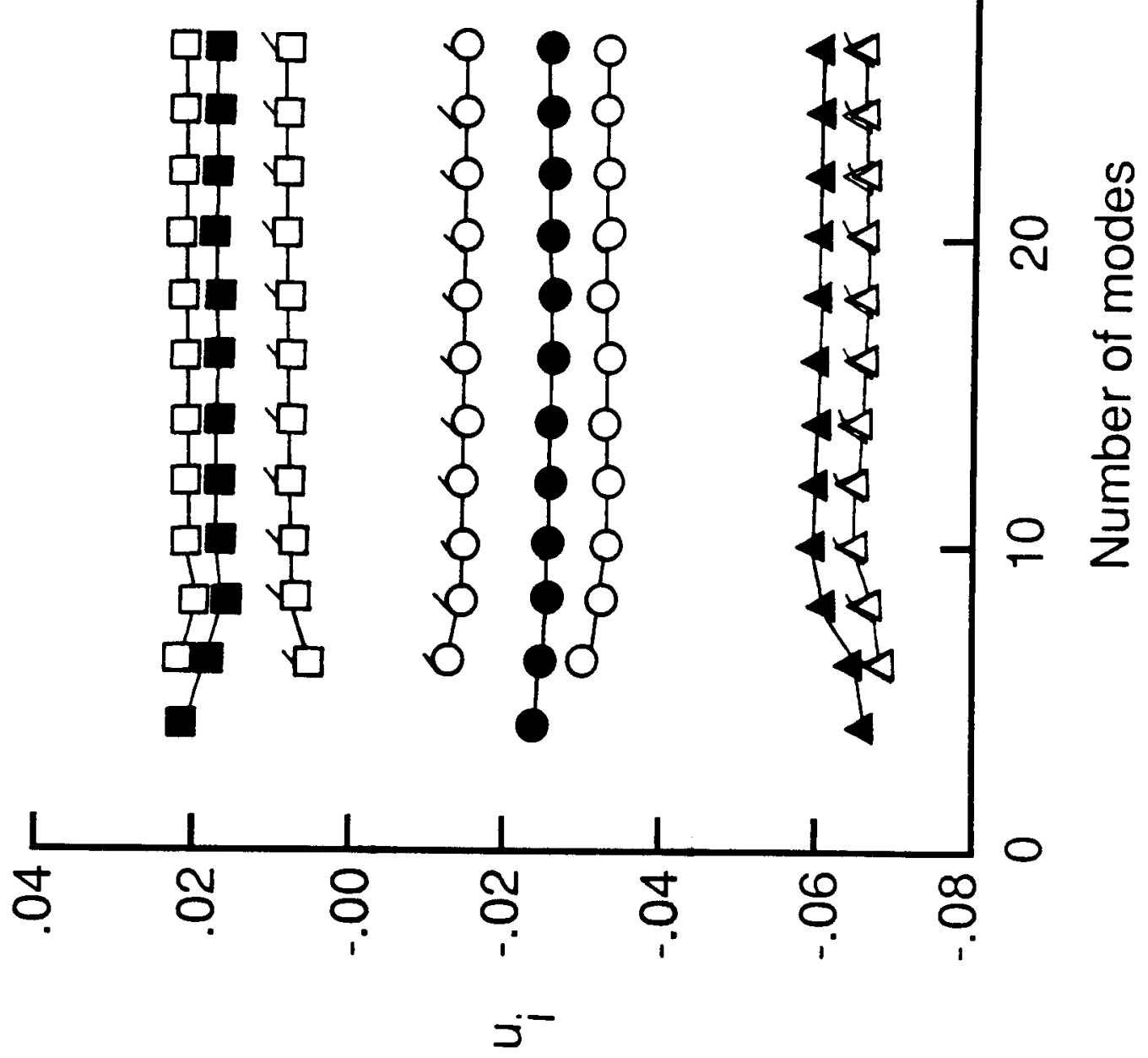

ह 

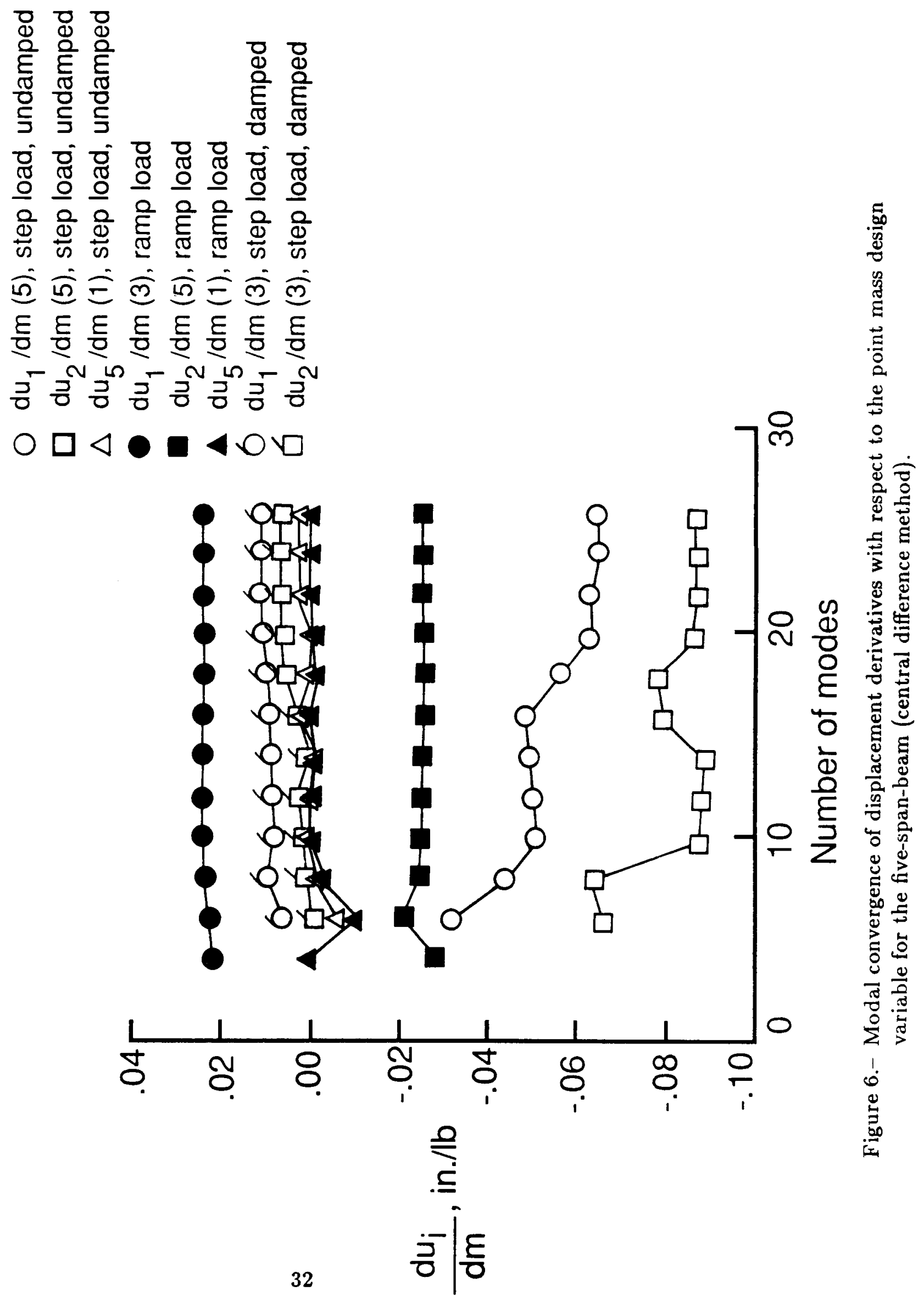

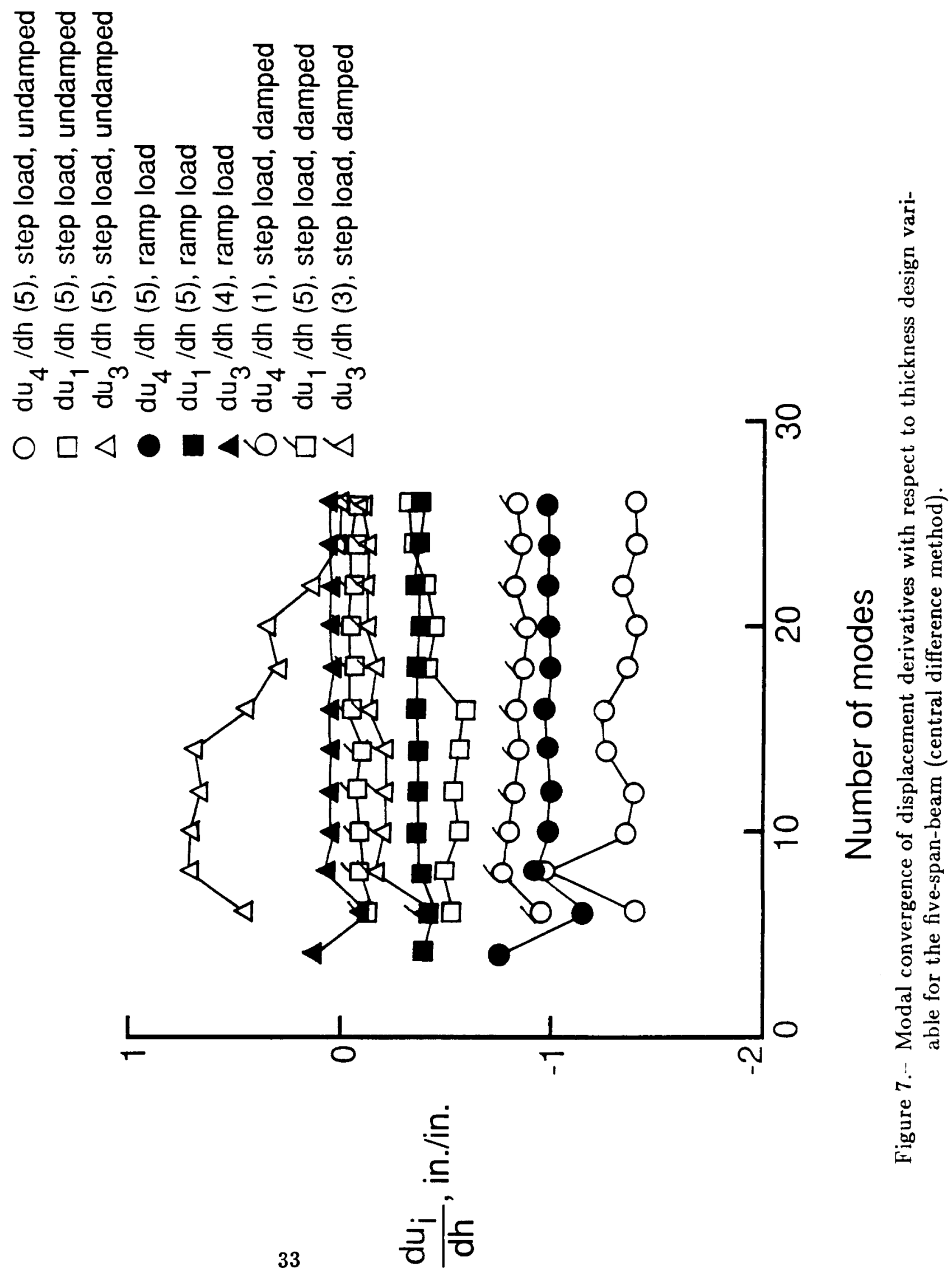

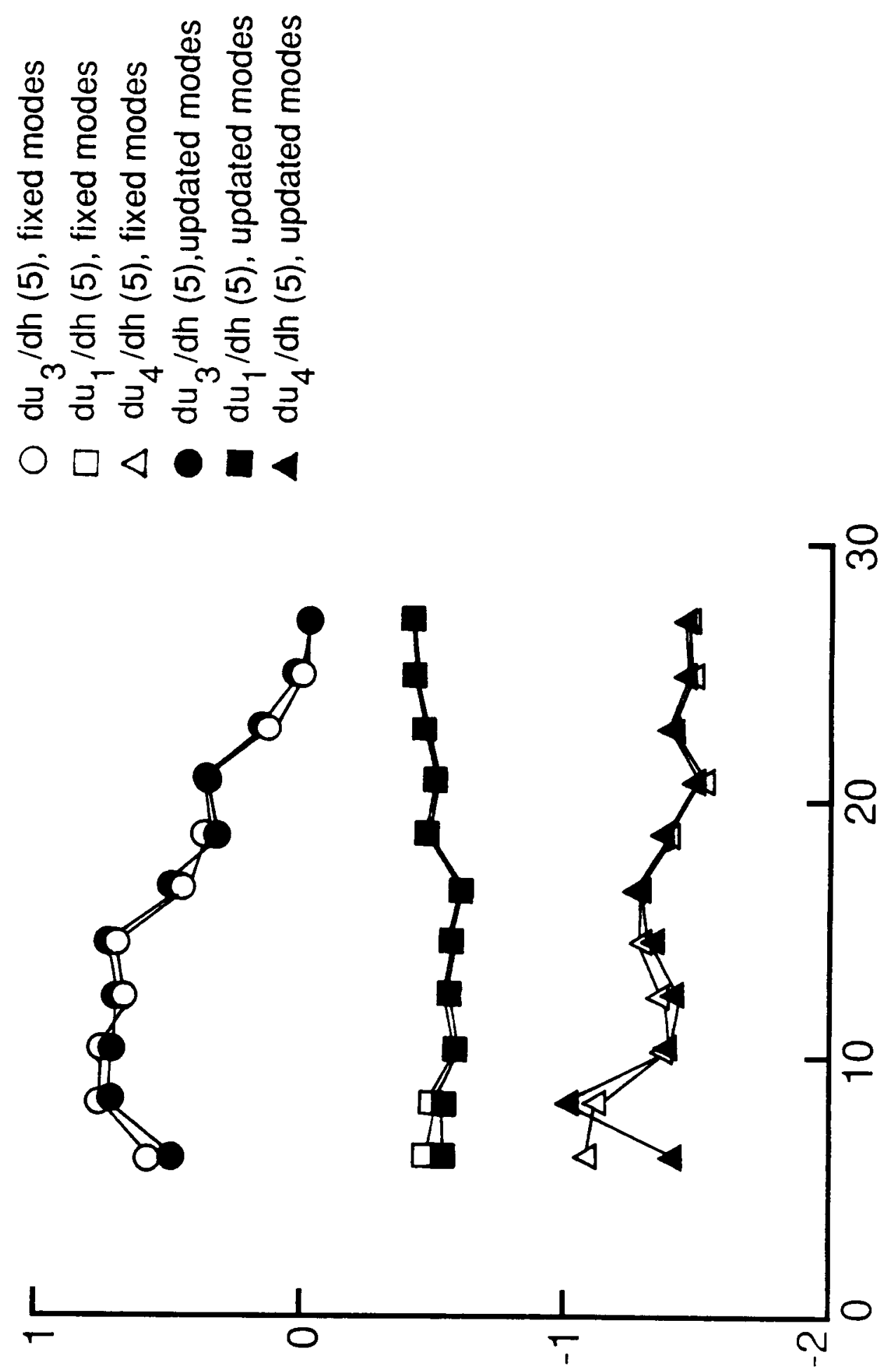

\begin{tabular}{l}
3 \\
$\frac{1}{8}$ \\
\hline \\
\hline \\
\hline
\end{tabular}
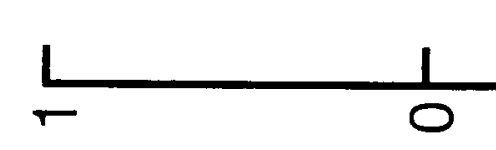

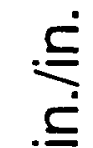

$$
\begin{aligned}
& \text { 흥 }
\end{aligned}
$$




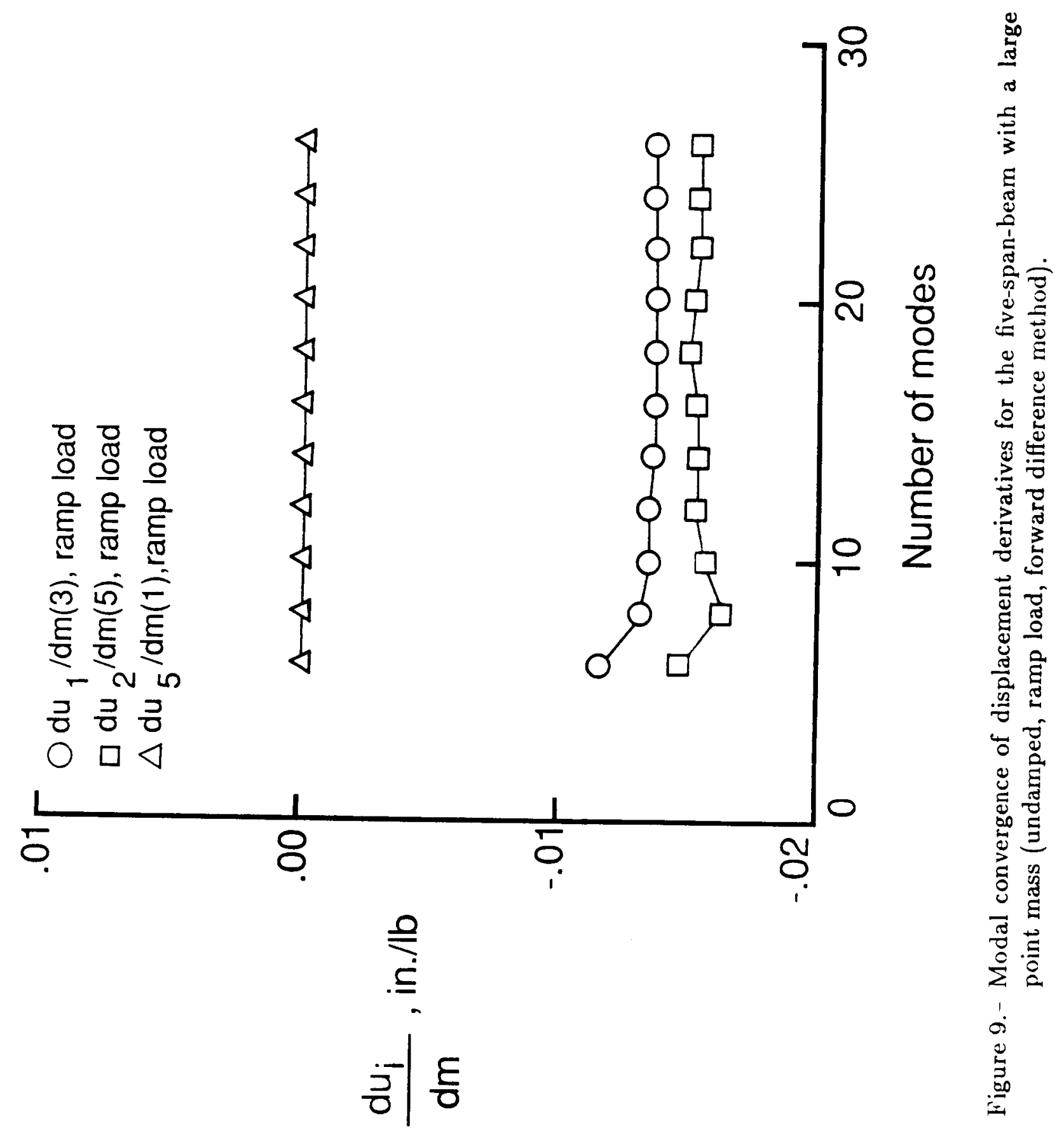




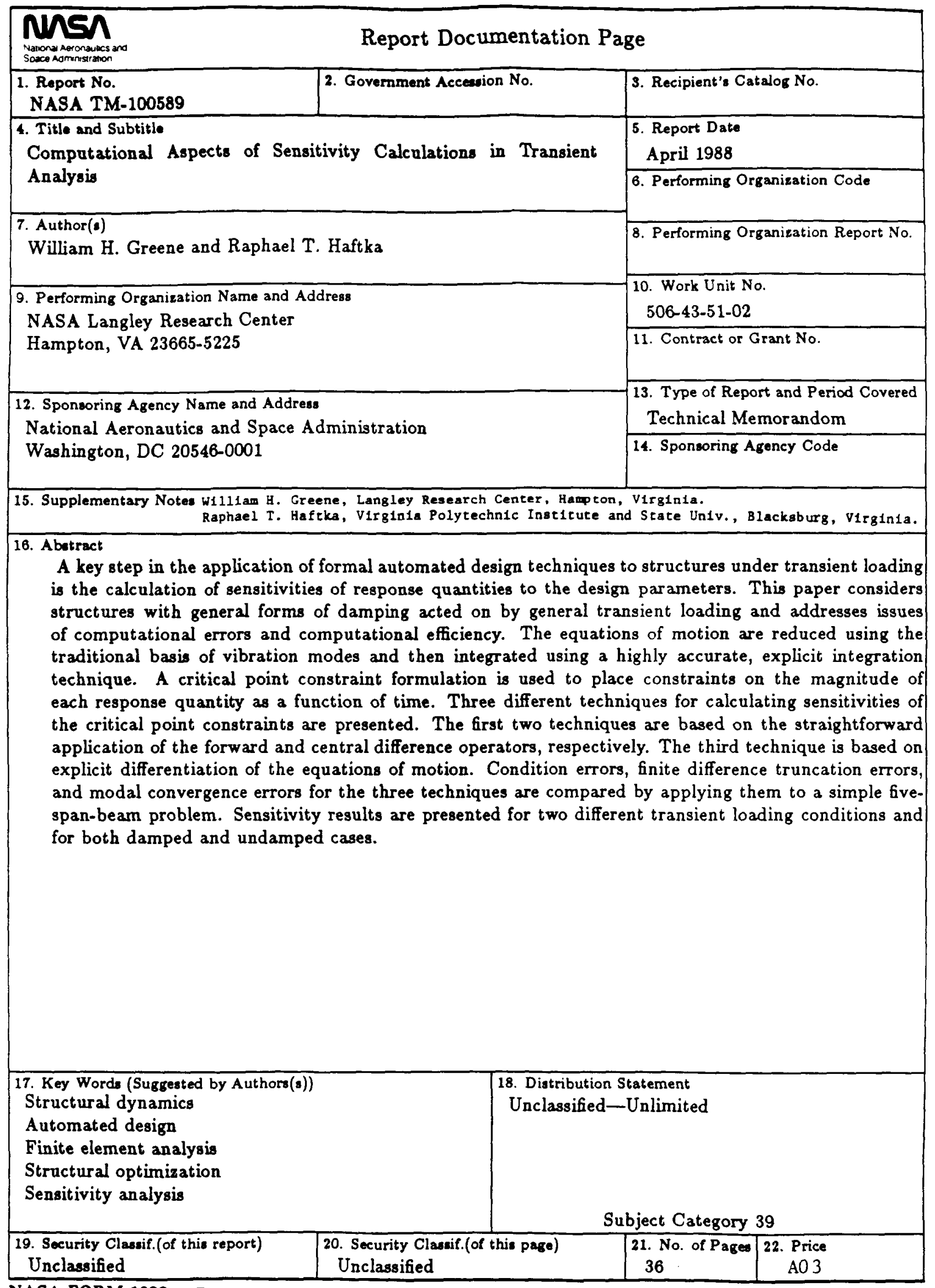

NASA FORM 1626 OCT 86

For sale by the National Technical Information Service, Springfield, Virginia 22161-2171 